\title{
Riscos à Saúde de Equipe de Enfermagem em Unidade de Terapia Intensiva: Proposta de Abordagem Integral da Saúde*
}

\author{
Risks to Health of Intensive Care Unity Nursing Staff: \\ Proposal of Integral Approach of Health
}

\author{
Érique José Peixoto de Miranda ${ }^{1}$, Kátia Stancato ${ }^{2}$
}

\section{RESUMO}

JUSTIFICATIVA E OBJETIVOS: O objetivo deste estudo foi rever a saúde ocupacional em unidade de terapia intensiva (UTI), bem como avaliar os aspectos para abordagem educacional da equipe multidisciplinar de forma integral.

CONTEÚDO: Revisão da literatura realizada no período de 1997-2007, acerca de questões relevantes da saúde de profissionais da equipe multidisciplinar de UTI, na base de dados da Biblioteca Regional de Medicina (Bireme). Foram utilizados os termos "UTI", "educação em saúde" e "saúde ocupacional", sem haver seleção por desenho de estudo.

CONCLUSÕES: Todos os estudos observados demonstraram que o ambiente de UTI é insalubre. Contudo entre os fatores que contribuem para tal insalubridade estão atitudes e hábitos dos profissionais de saúde da UTI, os quais são perfeitamente passíveis de mudança, razões pela qual uma abordagem de educação em saúde seria benéfica para diminuir o problema. As estratégias de educação continuada são adequadas na prevenção, tanto de riscos ocupa-

1. Graduando em Medicina pela Universidade Estadual de Campinas. 2. Professora Doutora do Departamento de Enfermagem da FCM da UNICAMP

*Recebido do Departamento de Enfermagem da Faculdade de Ciências Médicas da Universidade Estadual de Campinas (FCM - UNICAMP), Campinas, SP

Apresentado em 28 de agosto de 2007

Aceito para publicação em 12 de fevereiro de 2008

Endereço para correspondência:

Dra. Kátia Stancato - Faculdade de Ciências Médicas

R: Tessália Vieira de Camargo, 126

Cidade Universitária "Zeferino Vaz"

13083-970 Campinas, SP

E-mail: katia@fcm.unicamp.br; erique@fcm.unicamp.br

(C)Associação de Medicina Intensiva Brasileira, 2008 cionais quanto ambientais nas UTI.

Unitermos: educação em saúde, enfermagem, saúde ocupacional, UTI

\section{SUMMARY}

BACKGROUND AND OBJECTIVES: In this study we discuss about risks to health of intensive care unity staff and suggest a proposal of integral approach of health. CONTENTS: Literature review, from 1997 to 2007, at Bireme database about "health education", "intensive care unity", "nursing" and "occupational health", regardless of design of study.

CONCLUSIONS: All studies show that the environment of intensive care unity is unhealthy, which is also due to habits and attitudes of ICU health professionals. An approach to health education would be beneficial to minimize the problem. Strategies for continuing education are appropriate both in the prevention of occupational and environmental risks in intensive care units.

Key Words: health education, ICU, nursing, occupational health

\section{INTRODUÇÃO}

Serão discutidos aspectos da saúde ocupacional em unidade de terapia intensiva (UTI). Também serão avaliados os aspectos para abordagem educacional integral da equipe multidisciplinar, a qual lida com pacientes terminais e recursos tecnológicos sofisticados, embora se encontre, muitas vezes, desprovida de suporte emocional para lidar com o sofrimento alheio e consigo própria.

O objetivo deste estudo foi rever a saúde ocupacional em UTI, bem como avaliar os aspectos para abordagem educacional da equipe multidisciplinar de forma integral.

Para a abordagem do tema foi revista a literatura a respeito de questões relevantes de saúde de profissio- 
nais de equipe multidisciplinar de unidade de terapia intensiva (UTI) na base de dados on-line da Biblioteca Regional de Medicina (Bireme) (acesso em 28/7/2007). Foram usados na pesquisa os descritores "UTI", "educação em saúde" e "saúde do trabalho", no período de 1997 a 2007. Foram avaliados todos os trabalhos obtidos, sem ter sido efetuada seleção pelo desenho.

\section{HISTÓRICO}

As unidades de terapia intensiva constituem locais onde se internam pacientes graves, em situação limite, que ainda têm um prognóstico favorável para viver, embora necessitem de recursos técnicos e humanos especializados para sua recuperação; um ambiente onde são utilizados técnicas e procedimentos sofisticados, para tratar doenças com risco potencial à vida ${ }^{1}$.

Conforme os critérios da Society of Critical Care Medicine, os critérios de internação em UTI incluem doenças cardiovasculares, neurológicas, respiratórias, gastrintestinais, intoxicações, endocrinológicas, cirúrgicas (trauma e queimaduras graves) e infecciosas ameaçadoras à vida, bem como sinais vitais indicativos de gravidade (pulso $<40$ ou $>150$ batimentos por minuto, pressão arterial sistólica $<80 \mathrm{mmHg}$ ou 20 $\mathrm{mmHg}$ abaixo do nível habitual, pressão arterial média < $60 \mathrm{mmHg}$, pressão arterial diastólica > $120 \mathrm{mmHg}$, freqüência respiratória $>35 \mathrm{bpm}$ ); exames laboratoriais (níveis séricos de sódio $<110 \mathrm{mEq} / \mathrm{L}$ ou $>170 \mathrm{mEq} / \mathrm{L}$, níveis séricos de potássio $<2 \mathrm{mEq} / \mathrm{L}$ ou $>7 \mathrm{mEq} / \mathrm{L}$, $\mathrm{PaO} 2<50 \mathrm{mmHg}, \mathrm{pH}<7,1$ ou $>7,7$, glicose $>800 \mathrm{mg} /$ $\mathrm{dL}$, cálcio sérico $>15 \mathrm{mg} / \mathrm{dL}$, níveis tóxicos de drogas ou substâncias químicas em paciente hemodinamica ou neurologicamente comprometido); exames de imagem constatando hemorragia no sistema nervoso central ou contusão em pacientes com alteração do nível de consciência, sinais de rupturas de vísceras e vasos com instabilidade hemodinâmica.

Os critérios de alta incluem estabilização do quadro do paciente de tal forma que sua permanência na UTI não é necessária nem benéfica².

O ambiente das UTI é insalubre, onde a falta de treinamento e de precaução dos profissionais que trabalham nesse setor pode resultar em transmissão de doenças infecto-contagiosas e em acidentes ${ }^{3}$.

No Brasil, a preocupação com a questão da saúde dos trabalhadores hospitalares iniciou-se na década de 1970, quando pesquisadores da Universidade de São Paulo (USP) enfocaram a saúde ocupacional em trabalhadores hospitalares ${ }^{5}$. Esse estudo citou a ocor- rência de 4.468 acidentes de trabalho em hospitais do país ${ }^{5}$. Queixas como doenças infecto-contagiosas, lombalgias, reações alérgicas, fadigas e acidentes do trabalho foram referidos por 26 grupos ocupacionais de trabalhadores hospitalares em $1977^{6}$, ao passo que, em 1988, estudo realizado no Hospital das Clínicas da Universidade de São Paulo, envolvendo 1.506 acidentes levantou que as principais causas de afastamento foram lacerações e ferimentos, contusões ou torções ${ }^{7}$. Entre os nexos referidos na literatura pode-se citar lombalgias relacionadas ao transporte e movimentação de pacientes, bem como a transmissão ocupacional de doenças infecciosas, genitourinárias, psicossomáticas, cardiovasculares e osteomusculares relacionadas ao contato com pacientes e ao exercício dos procedimentos ${ }^{8-11}$

Apesar do relatado, os trabalhadores da área da saúde não eram considerados como categoria profissional de alto risco para acidentes do trabalho, fato que mudou a partir do surgimento do vírus da imunodeficiência humana (HIV) e da síndrome da imunodeficiência adquirida (SIDA) como epidemia nos anos $1980^{2}$. Foram introduzidas, então, pelo Center for Disease Control and Prevention (CDC), dos Estados Unidos, as "precauções universais", atualmente denominadas "precauções padrão", que alertam a necessidade da utilização de luvas para o contato com fluidos corporais ${ }^{11}$.

O exercício da enfermagem está associado à exposição contínua a riscos biológicos ${ }^{13}$. O contato direto com o paciente durante a assistência e os procedimentos, sobretudo punção venosa periférica (30\%-35\%) $)^{14}$ expõe os trabalhadores, principalmente a equipe de enfermagem, ao risco destas infecções através de ferimento percutâneo ou contato de membrana, mucosa ou pele com sangue ou outros fluidos corpóreos potencialmente infectados ${ }^{11}$.

Devido à falta de medidas de proteção coletiva, tornase necessário o uso de equipamento de proteção individual, destinado a proteger a saúde e a integridade física do trabalhador. Nesse equipamento são incluídas luvas, aventais, protetores oculares, faciais e auriculares, protetores respiratórios e de membros inferiores ${ }^{15}$. A partir da década de 1980, profissionais da área da saúde voltaram suas atenções ao estudo das repercussões do processo de trabalho hospitalar como causador de doenças e acidentes em seus trabalhadores e usuários $^{3}$. Entretanto, somente na década de 1990, foram levados em conta aspectos éticos e psíquicos do trabalho na área de saúde. Apesar desse fato, as doenças ou queixas não relacionadas ao trabalho continu- 
am sujeitas a uma análise mais apurada para exclusão de seu nexo causal com o processo de trabalho ${ }^{3}$. Um estudo de caso-controle realizado com 1.218 trabaIhadores de enfermagem de um hospital universitário constatou a incidência acumulada de $8,2 \%$ acidentes de trabalho ${ }^{16}$. Esses acidentes estavam associados à falta de tempo para lazer e às posturas cansativas e forçadas durante o trabalho, sobretudo por parte da equipe de enfermagem, bem como a esquemas vacinais incompletos (dupla adulto e hepatite B) dos trabaIhadores. A autora desse trabalho sugeriu que deveriam ser elaborados programas de vigilância de saúde no trabalho, campanhas de vacinação e de combate ao alcoolismo e destacou como fatores de risco para os acidentes a condição sócio-econômica, a idade e as condições físicas do empregado, bem como o ambiente de trabalho e as instalações ${ }^{16}$. Um estudo ${ }^{17}$ que aponta os principais riscos ocupacionais aos quais os trabalhadores de UTI estão expostos e traça medidas de proteção específica que vão desde a planta física até o preparo técnico da equipe.

\section{EDUCAÇÃO EM SAÚDE}

A abordagem integral da saúde da equipe multidisciplinar de uma UTI deve levar em conta os riscos ambientais e ocupacionais aos quais os profissionais dessas unidades estão expostos diariamente. São riscos ambientais aos agentes físicos, químicos e biológicos existentes no ambiente de trabalho, que, dependendo da sua natureza, concentração ou intensidade e tempo de exposição, são capazes de causar lesões à saúde dos trabalhadores ${ }^{18}$. Riscos ocupacionais são todas as situações de trabalho que podem romper o equilíbrio físico, mental e social das pessoas, e não somente as situações que originem acidentes e doenças ${ }^{15}$.

Entre os métodos de controle desses riscos estão a substituição do agente de risco, controles de engenharia, práticas de trabalho, equipamentos de proteção pessoal, controles administrativos e programas de exames médicos periódicos ${ }^{19}$.

A abordagem integral da prevenção de acidentes de trabalho pode ser feita pela educação em saúde, uma prática social ou processo que contribui para a formação e desenvolvimento da consciência crítica das pessoas, a respeito de seus problemas de saúde e estimula a busca de soluções e a organização para a ação coletiva. Não se resume a um processo de persuasão ou de transferência de informação, sendo também um processo de capacitação para transformar a realidade.
A história da educação em saúde tem como característica um processo de mudanças, o qual se faz presente na própria nomenclatura desta área, denominada na segunda metade do século XIX de "educação higiênica", devido à associação à revolução bacteriana, passando à "educação sanitária" nos anos 1920, com o desenvolvimento da saúde pública que enfatiza processos de prevenção, mas ainda norteada por uma orientação comportamentalista. Finalmente na década de 1970 a "educação em saúde", incorporou os aspectos sócio-econômicos e culturais ${ }^{20}$.

A atual educação em saúde interpreta os processos de saúde e doença a partir de vários referenciais e privilegia práticas participativas, considerando que os educadores e a população têm saberes que se interagem, sendo unidos na "luta" por melhores condições de vida, superando os limites da ação sanitária para alcançar uma ação social de mudanças, comprometida com a promoção da saúde e o com o bem-estar geral. Entre as abordagens propostas para essa prática está a humanista (o homem é arquiteto de si mesmo), a comportamentalista (o homem é fruto do seu meio e o ambiente social dá forma e preserva o comportamento; a experiência é a base do conhecimento) e político-social (os seres humanos estão em constante interação com o meio, são agentes da transformação deste e sujeitos da sua própria educação) ${ }^{21}$.

Entre os modelos propostos para a intervenção no meio está o de mudança de comportamento (o especialista acha, dentro de uma concepção biomédica, o que o cliente deve fazer; a prevenção tem nível primário [informação e prevenção], secundário [detecção e tratamento de doenças] e terciário [doenças terminais e crônicas]), o modelo de autofortalecimento (aprendizagem participativa, de orientação humanista), o modelo de orientação comunitária (o indivíduo é responsável pela própria saúde) e o de transformação social (mudança social através de movimentos coletivos organizados; as mudanças no nível das idéias necessitam desmistificar e tornar acessível o conhecimento médico separando-se os argumentos científicos das considerações morais) ${ }^{21}$. A educação em saúde faz parte de uma reformulação do sistema de saúde que extrapola o âmbito administrativo, a qual deve ser estendida ao melhoramento da relação entre instituição, profissional de saúde e usuários no afã de possibilitar a reeducação de ambos os agentes, o técnico e o usuário, tendo por referência o desenvolvimento da cidadania social e política e a busca da construção de sujeitos, com respeito à autonomia e à emancipação ${ }^{22}$ 


\section{RISCOS OCUPACIONAIS}

A UTI é um lugar de tensões constantes, que responde ao desafio da saúde com divisão do trabalho transformando as emergências em rotina, onde profissionais experimentam uma vivência de extrema angústia, algo que parece ser pior que a morte, a qual, freqüentemente, não se leva em consideração ${ }^{1}$. Trata-se de um medo próprio da precariedade da existência humana, experiência revestida de dificuldades; a experiência da morte do próximo faz surgir a consciência do que seja morrer ${ }^{1}$.

Com relação aos dilemas éticos sobre o fim da vida, Moritz demonstrou que a recusa ou a suspensão de tratamentos foram constatados em $32 \%$ dos óbitos em UTI de um hospital universitário e a futilidade da terapia foi considerada como principal motivo em $100 \%$ destes $\operatorname{casos}^{23}$.

O estresse e outras conseqüências biopsicofisiológicas às quais os profissionais de uma UTI estão expostos, de forma cumulativa e progressiva, são desencadeados por fatores como ambiente de trabalho, sobrecarga de trabalho, relações interpessoais, trabalho noturno, tempo de serviço (intrínsecos) e condições pessoais e características da personalidade (extrínsecos), conforme estudo que levou em conta o problema entre enfermeiros brasileiros de 1982 a $2001^{24}$. Outros agentes estressores apontados foram a organização do trabalho, sobretudo em ambiente com precariedade das condições laborais, o ambiente ruidoso, as relações conflitantes e as exigências impostas pelo trabaIho ${ }^{25}$. A importância da identificação desses agentes estressores, principalmente em uma abordagem de educação em saúde e preventiva, consiste em perspectivas para um ambiente de trabalho seguro, o que pode gerar motivação e diminuir os riscos aos quais o grupo está exposto ${ }^{26}$.

Apesar de sofrimento, o trabalho em saúde é uma fonte de prazer ${ }^{27}$. Gomes e col. quantificaram como agentes estressores o rígido controle do tempo; forma como o setor é organizado; falta de materiais e equipamentos adequados; conflitos nos relacionamentos entre os membros da equipe; estado crítico de saúde do paciente; dupla jornada de trabalho feminino (a dupla jornada é também pela má remuneração e tanto homens como mulheres estão expostos); e trabalho nos finais de semana e feriados ${ }^{26}$. Para minimizar os sentimentos negativos com relação ao exercício profissional Gomes e col. sugeriram que se criem espaços para discussões coletivas acerca da gênese do sofrimento psíquico no trabalho, de forma que os profissionais se comprometam com a melhoria da saúde ocupacional e organização do trabalho ${ }^{26}$.

Em estudo descritivo realizado na UTI pediátrica do Instituto Fernandes Figueira, Rio de Janeiro, no período de 2002 a 2003, constatou-se que entre os profissionais com faixa etária de 20 a 50 anos, $90 \%$ dos quais do sexo feminino, com predominância de jovens de 20 a 29 anos - 60\% tinham dupla e tripla jornada, com carga horária acima de 60 horas semanais, estilo de vida não saudável em relação ao lazer, exercício físi$\mathrm{co}$, repouso e sono ${ }^{27}$. Os riscos ocupacionais percebidos foram ritmo acelerado no trabalho, manutenção de posturas inadequadas, esforço físico que produz fadiga, trabalho isolado, temperatura inadequada, excesso de ruído, exposição à irradiação e risco de infecção. As doenças relacionadas com as condições de trabalho foram: distúrbios osteomusculares, varizes e estresse. Concluiu-se que os problemas de saúde e condições de trabalho estão inter-relacionados e é impossível isolar causa e efeito ${ }^{28}$.

Em outro serviço da mesma cidade, estudo com 47 profissionais mostrou três categorias teóricas que justificavam o estresse: os riscos ocupacionais decorrentes do trabalho de enfermagem, as conseqüências da exposição do trabalhador às cargas de trabalho e o prazer e o sofrimento como expressão do trabalho cotidiano. Concluiu-se que a UTI é um ambiente repleto de riscos e que a equipe de enfermagem se defronta com diferentes cargas de trabalho, com o sofrimento e o prazer no desenvolvimento de suas atividades cotidianas $^{29}$.

Trata-se, portanto, de um ambiente estressante. Em estudo descritivo em UTI de hospital universitário no Brasil, constatou-se uma prevalência de $59,4 \%$ de estresse ocupacional entre profissionais de Enfermagem, os quais mencionaram como causas a gravidade dos pacientes e a instabilidade do quadro clínico, o atendimento de parada cardiorrespiratória e das emergências, bem como os riscos por sistema hemodialítico e o risco biológico do contato com sangue e secreções ${ }^{5}$.

Em outro estudo, com participação de 132 funcionários de UTI, $60 \%$ indicaram que o seu trabalho é estressante e que se sentiam fisicamente fatigados, $60 \%$ declararam que o seu trabalho teve impacto negativo sobre a vida familiar e $35 \%$ indicaram que a sua saúde estava afetada e apenas $51 \%$ indicaram que planejavam continuar trabalhando em UTI ${ }^{30}$. As relações entre os membros da equipe foram relatadas como boa por $90 \%$.

Em trabalho realizado com 144 profissionais de tera- 
pia intensiva, idade média de 32 anos e média de 8 anos de experiência, $41 \%$ tinham desenvolvido transtorno de estresse pós-traumático, sobretudo devido a uma média de 38 experiências traumáticas como sua própria ansiedade e desamparo, além do contato com pessoas gravemente feridas e/ou mutiladas, pacientes terminais e cadáveres ${ }^{30}$.

Tendo isso em vista, o objetivo em uma intervenção ideal consiste em fomentar mecanismos eficazes para reduzir o estresse, diminuir a rotatividade de funcionários, implementar estratégias para valorizar a profissão, sem deixar de investir na qualidade da assistência ao paciente e na segurança ${ }^{31}$. Uma estratégia amplamente indicada para casos de estresse ocupacional é o Critical Incident Stress Debriefing (CISD), como parte de uma abordagem multidimensional no manuseio do problema $^{32}$.

Além do estresse, outro risco ocupacional citado na literatura é a agressividade física e/ou verbal dos pacientes, parentes e visitantes das UTI. Em estudo brasileiro $4 \%$ dos trabalhadores mencionaram terem sido agredidos $^{4}$, ao passo que, em inquérito realizado na Inglaterra e no País de Gales, o abuso verbal cometido por pacientes e parentes contra enfermeiros ocorreu, respectivamente, em $87 \%$ e $74 \%$ das UTI. Contra médicos, os números são, respectivamente, $65 \%$ e $59 \%$. As principais causas associadas à agressão apontadas no estudo são distress (45\%), álcool (24\%) e comportamento sociopata $(27 \%)^{33}$. Os autores admitem que o inquérito provavelmente subestima o problema e aponta como tentativa de intervenção um investimento em sistemas de segurança (presente em 43\% das UTI do estudo), sensibilização dos profissionais e formação em habilidades de comunicação. Em outro trabalho, 54\% experimentaram eventos ameaçadores ou violentos; $83,8 \%$ sentiram-se confiantes em sua capacidade de reconhecer situações violentas; $69,1 \%$ sentiram-se confiantes em sua capacidade de gerir situações violentas e, embora houvesse diversos meios de notificação desses acontecimentos, $27 \%$ não os relataram nem os documentaram ${ }^{34}$. Programas educacionais bem sucedidos para combater o problema são citados na literatura ${ }^{35}$.

\section{RISCOS BIOLÓGICOS}

Os riscos ocupacionais da equipe intensivista estão inter-relacionados com os riscos de seus pacientes, os quais, em investigação diagnóstica devido a doenças diversas, passam por um elevado número de proce- dimentos e intervenções terapêuticas que necessitam utilizar materiais pérfuro-cortantes e expõem profissionais de saúde ao contato com sangue, secreções, fluidos corpóreos por incisões, sondagens e cateteres ${ }^{4}$. São os riscos mais freqüentes aos quais os trabalhadores estão expostos ${ }^{4,36-38}$.

As atividades de arranjo do ambiente após os procedimentos, encaminhamento dos materiais, limpeza e a organização da unidade do paciente são as atividades relacionadas a acidentes com materiais pérfurocortantes ${ }^{4}$.

Os agentes mais importantes de transmissão parenteral são o vírus da hepatite $B(\mathrm{HBV})$, hepatite $\mathrm{C}(\mathrm{HCV})$ e imunodeficiência adquirida humana (HIV). O risco de hepatite B clínica varia entre $22 \%$ a $31 \%$ e o da evidência sorológica de infecção de $37 \%$ a $62 \%$, quando há exposição percutânea ao sangue de paciente $\mathrm{HBe}$ Ag positivo ${ }^{39}$. Se HBeAg negativo, o risco de hepatite clínica varia de $1 \%$ a $6 \%$ e o de soroconversão, $23 \%$ a $37 \%$. Quanto à hepatite $\mathrm{C}(\mathrm{HCV})$, transmitida de forma eficiente através do sangue, a incidência média de soroconversão, após exposição percutânea com sangue sabidamente infectado pelo HCV, é de 1,8\% (IC 95\% de $0 \%-7 \%)^{39}$. O risco de transmissão em exposições a outros materiais biológicos e por exposições de mucosas é considerado mais baixo. Nenhum caso de contaminação envolvendo pele não-íntegra foi publicado na literatura. Menos freqüente, o risco de transmissão do HIV é de 0,3\% (IC 95\%=0,2-0,5\%) em acidentes percutâneos e de 0,09\% (IC 95\%=0,006-0,5\%) após exposições em mucosas ${ }^{39}$. Estima-se que o risco após exposições envolvendo pele não-íntegra seja inferior ao risco das exposições em mucosas.

Em estudo descritivo $69 \%$ dos trabalhadores disseram estarem expostos a materiais pérfuro-cortantes ${ }^{4}$. Seis por cento deles admitiram descartá-los em locais inadequados. O uso de lâminas em atividades não necessárias e o abandono de material descartável usado em lugares inadequados correlacionaram-se com o aumento do número de acidentes ${ }^{4}$.

Entre os fatores associados à redução da taxa de acidentes estão a monitorização do trabalho de enfermeiros, as melhorias das condições de trabalho, da organização dos hospitais e do cuidado dispensado aos pacientes e a baixa rotatividade de funcionários ${ }^{40}$. Nesse estudo multicêntrico norte-americano que envolveu 39 UTI de 23 hospitais, houve correlação significativa entre lucratividade e diminuição de acidentes pérfurocortantes e exposição a fluidos biológicos $(p<0,05)^{40}$. $\mathrm{O}$ uso de dispositivos seguros (safety-engineered de- 
vices) em procedimentos também está associado à redução de acidentes. Um estudo multicêntrico francês envolvendo 32 hospitais e 1.506 médicos e enfermeiros de UTI, entre 1999 e 2000, em seguimento de um ano, obteve como resultado uma correlação positiva entre diminuição de acidentes e aumento do uso desses dispositivos $(r=0,88 ; p<0,02)$. A taxa média de acidentes no trabalho foi estimada em 4,72 casos por 100.000 procedimentos $^{41}$.

Além desses agentes, os profissionais estão expostos a doenças de transmitidas por gotículas, aerossóis e contato direto, tais como tuberculose, citomegalovirose, rubéola, meningite, difteria, herpes simples, herpes zoster, febre tifóide, gastroenterite infecciosa, parotidite e ceratoconjuntivite epidêmica e infecções respiratórias por vírus, citando ainda as doenças causadas por bactérias envolvidas nas infecções hospitalares, tais como Staphylococcus aureus, Escherichia coli, Salmonella spp., Streptococcus spp., Pseudomonas spp., Proteus spp. ${ }^{42}$.

A tuberculose, devido à elevada incidência no Brasil, constitui um motivo de grande preocupação entre os trabalhadores da área saúde. A transmissão em hospitais envolve pacientes com doença pulmonar ou laríngea não diagnosticada, sem esquema terapêutico nem isolamento respiratório, e profissionais durante procedimentos como broncoscopia, intubação e aspiração traqueal, irrigação de abscesso ou qualquer outro que estimule a tosse, sobretudo em ambiente fechado.

\section{RISCOS ERGONÔMICOS}

A ocorrência dos distúrbios osteomusculares relacionados ao trabalho (DORT) vem crescendo nas últimas décadas, risco ao qual os profissionais de saúde estão expostos devido à movimentação e ao transporte de pacientes, que exigem grande esforço físico e estão associados a problemas músculo-esqueléticos.

Os trabalhadores de enfermagem em UTI desenvolvem muitas atividades que exigem esforço físico, tais como manusear o paciente, retirar e colocar monitores de prateleiras e mesas auxiliares, organizar os equipamentos e mobiliário à beira do leito e em salas especiais, dispor materiais de consumo no posto de trabalho e separar os equipamentos e mobiliários com problemas técnicos para reparos ${ }^{4}$.

Em estudo realizado em 2003, em hospital universitário, por meio de escala de avaliação do risco na movimentação e transferência, que apresenta propriedades psicométricas confiáveis, demonstrou-se que a UTI é a unidade que apresentou o maior percentual de pacientes $(64 \%)$ os quais oferecem alto risco ergonômico aos trabalhadores ${ }^{42}$. As unidades cirúrgicas apresentaram pacientes oferecendo médio risco. Os pacientes que ofereceram pouco risco ergonômico estavam, em sua maioria, em unidades clínicas ${ }^{43}$.

A manutenção de posturas inadequadas no transporte de pacientes e uma distribuição de tarefas que acarreta sobrecarga, uso em UTI de camas com dispositivo manual de ajuste, macas sem ajuste de altura, monitores com parâmetros e alarmes insuficientes, ausência de equipamentos para mobilização e transferência de pacientes são fatores que acabam contribuindo para lesões por esforço físico ${ }^{3}$. Esses dados são corroborados também por Stucke e Menzel ${ }^{44}$.

Além das causas citadas, a falta de espaços adequados para tarefas dos profissionais na UTI constitui um risco ergonômico. Hignett e Lu constataram empiricamente uma necessidade média de $23,26 \mathrm{~m}^{2}$ para transferência de pacientes de cama a cama e 22,87 $\mathrm{m}^{2}$ para reanimação. A média de espaço necessário para funções de cuidado foi $22,83 \mathrm{~m}^{2}{ }^{45}$.

\section{RIScos Físicos}

Entre os riscos físicos citados na literatura, destacamse a exposição à radiação e a ruídos, bem como problemas decorrentes de instalação elétrica, iluminação e climatização.

Em estudo realizado em UTI de hospital universitário, $22 \%$ dos trabalhadores citaram a exposição à radiação como um risco existente no ambiente de trabalho ${ }^{3}$. A exposição é diária e periódica, devido a procedimentos radiológicos de rotina no leito (localização de cateteres, fraturas e arteriografias), porém não contínua. Entretanto um estudo internacional em UTI de centro de trauma não confirmou a exposição à radiação como risco ocupacional, uma vez que os 15 dosímetros instalados na UTI detectaram um nível de radiação inferior ao valor recomendado de menos de 100 mrem (millrröentgen) por $a^{4}{ }^{46}$. A despeito disso, outro estudo brasileiro demonstrou que os profissionais de UTI estão expostos à radiação ionizante por não estarem cientes das recomendações previstas na legislação ${ }^{47}$.

Quanto aos ruídos, apesar de freqüentes e contínuos no ambiente de UTI, dada a presença dos variados tipos de alarmes integrados aos modernos equipamentos, são pouco percebidos pelos trabalhadores como risco para a sua saúde. Estudo refere que apenas 3\% trabalhadores mencionaram esse fator como risco am- 
biental ${ }^{4}$. Outro estudo brasileiro concluiu que o nível basal de ruído em UTI variava entre 60 e $70 \mathrm{~dB}$, com nível máximo de $120 \mathrm{~dB}$, valores acima do que recomendam a Agência de Proteção Ambiental dos Estados Unidos (25 e $35 \mathrm{~dB}$ para os períodos noturno e diurno, respectivamente) e International Noise Council${ }^{48}$. Estudo grego encontrou variação de 60,3-67,4 dB ${ }^{49}$.

Embora não seja percebido, o nível de ruído em UTI correlaciona-se com estresse. Estudo que avaliou a freqüência cardíaca ( $F C)$ continuamente, bem como o aborrecimento referido em questionário e a amilase salivar a cada 30 minutos, encontrou correlação entre níveis sonoros elevados e FC alta $(p=0,014)$, estresse subjetivo $(p=0,021)$ e aborrecimento $(p=0,016)^{50}$. A média diurna de nível sonoro foi $61 \mathrm{~dB}(\mathrm{~A})$ e noturna, $59 \mathrm{~dB}(\mathrm{~A})$. Outros importantes preditores de taquicardia foram maior ingestão de cafeína, equipe de enfermagem menos experiente e período diurno ${ }^{50}$.

Em estudo realizado em UTI de Curitiba, constatou-se que dos 509 pacientes avaliados, 127 queixaram-se de barulhos na unidade, sendo: 1) barulhos em geral (alarmes, quedas de objeto-36,30\%); 2) conversa alta $(33,88 \%)$; 3$)$ pacientes gemendo $(16,93 \%) ; 4)$ telefone $(6,45 \%)$; 5$)$ porta batendo $(4,03 \%) ; 6)$ campainha $(2,41 \%)$. Da equipe avaliada $75 \%$ responderam o protocolo de investigação dos estressores, sendo: 1) alarmes (92\%); 2) barulho do respirador (84\%); 3) conversa alta (80\%); 4) telefone (64\%); 5) campainha (28\%); 6) pacientes gemendo (24\%). A média de ruído encontrada foi de $61 \mathrm{~dB}$, valor acima do recomendado no Brasil ${ }^{51}$.

A falta de conhecimento da equipe intensivista acerca do nível de ruído é também corroborada por outro estudo, o qual também avalia os poucos compreendidos efeitos da exposição, entre os quais a diminuição da cicatrização, a privação de sono e a estimulação cardiovascular em pacientes, bem como os problemas de desempenho cognitivo para os profissionais da UTI52.

Para combater a poluição sonora na UTI, sensibilidade e consciência pessoal são necessárias. Em estudo quase experimental que avaliou a mudança de comportamento da equipe evidenciou uma redução do nível de ruído de três a quatro vezes (de $L$ [min] 62-70 $\mathrm{dB}$ e L[max] 78-100 dB para 47-51 dB e 68-84 dB), respectivamente ${ }^{53}$.

Outros riscos apontados na literatura incluem quedas por piso liso e/ou molhado e arranjo físico inadequado, desconforto térmico, instalação elétrica e iluminação inadequadas, citadas por trabalhadores em questionário como fatores de risco de acidentes ${ }^{4,54}$. As normas para climatização de UTI estipulam temperatura mantida entre 24 e $26^{\circ} \mathrm{C}$, estável e igual em todos os compartimentos, evitando deslocamento de ar excessivo e conservando a umidade relativa em níveis de $40 \%$ a $60 \%$; a qualidade do ar deve ser segura, satisfatória e mantida estável durante todo o tempo, com no mínimo seis trocas de ar por hora, duas das quais devem ser com ar externo ${ }^{55}$.

\section{RISCOS QUÍMICOS}

Em estudo realizado em UTI de hospital universitário, obteve uma prevalência de $22 \%$ de exposição a agentes químicos, entre os quais produtos de limpeza e anti-sépticos, o que denota baixa percepção aos ris$\cos ^{4}$. Outro estudo constatou que os auxiliares de enfermagem estavam expostos a $35,9 \%$ e os enfermeiros a $28,3 \%$ do total de produtos químicos que constituem risco potencial ${ }^{56}$.

Um inquérito acerca de dermatite ocupacional de mãos encontrou prevalência de $55,6 \%$ no total da população e $69,7 \%$ nos profissionais mais expostos (freqüência de lavagem superior a 35 vezes por turno [odds ratio de 4,13, $p<0,005]$ ), o que pode representar um risco adicional em exposição a agentes biológicos. Não foi encontrada nenhuma relação entre dermatite e idade, sexo, etnia, atopia, história anterior de dermatite e duração do emprego ${ }^{57}$.

\section{EQUIPAMENTO DE PROTEÇÃO INDIVIDUAL}

Com relação ao uso de equipamento de proteção individual (EPI), em estudo realizado em hospital universitário brasileiro, encontrou-se uma prevalência de $4 \%$ de uso irregular de luvas, $29 \%$ de máscaras, $29 \%$ de avental e $84 \%$ de óculos em procedimentos ${ }^{4}$. Não houve diferença significativa entre as categorias profissionais. O motivo mais significativo para a irregularidade de uso foi a falta de hábito e/ou disciplina.

Em relação ao uso de óculos de proteção, alguns motivos foram alegados, entre eles inadequação do equipamento, quantidade insuficiente e o fato de usar óculos de grau.

Quanto ao uso das luvas, o motivo dos trabalhadores nem sempre utilizá-las esteve relacionado, principalmente, ao esquecimento e à inadequação do EPI. Esses motivos retratam a não valorização e a falta de conscientização sobre o uso de EPI como fator de proteção para os trabalhadores ${ }^{4}$.

Em outro trabalho descritivo, concluiu-se que os aci- 
dentes em UTI associaram-se à falta de recursos humanos e materiais, bem como a falta de conscientização dos enfermeiros na utilização de EPI ${ }^{58}$.

\section{CONCLUSÃO}

Estratégias de educação continuada dos funcionários quanto as medidas de precaução diante de agentes biológicos, físicos e químicos, reformulação de políticas de prevenção de riscos ocupacionais, avaliação contínua da saúde dos trabalhadores, dos ambientes hospitalares e elaboração de mapa de riscos, com base nos estudos citados, fazem parte de uma estratégia de intervenção para redução de riscos em UTI. Todos os estudos apresentados demonstraram que o ambiente de UTI é insalubre. Contudo entre os fatores que contribuem para tal insalubridade estão atitudes e hábitos dos profissionais de saúde da UTI, os quais são perfeitamente passíveis de mudança, razões pela qual uma abordagem de educação em saúde seria benéfica para diminuir o problema.

$\mathrm{O}$ investimento em recursos humanos e materiais, uso de EPI, atualização de esquemas vacinais, ênfase nas precauções de contato, gotículas e aerossóis de forma a evitar transmissão de doenças, medidas de redução do estresse ocupacional e da violência no ambiente de trabalho, bem como valorização da profissão e adequação de jornadas de trabalho também são fundamentais na melhoria das condições de trabalho e até mesmo no âmbito de administração hospitalar, haja vista a redução de despesas.

Sugere-se que em programa de educação continuada e saúde em UTI, após abordagem de temas relevantes em se tratando do ambiente de trabalho, também se dê atenção a problemas prevalentes nesse meio, diretamente ou indiretamente relacionados ao exercício da profissão, tais como diabete melito, hipertensão arterial, tabagismo, etilismo, uso de drogas ilícitas, obesidade, sedentarismo, doenças cardiovasculares, neoplasias e doenças mentais.

\section{REFERÊNCIAS}

01. Oliveira ECN - O psicólogo na UTI: reflexões sobre a saúde, vida e morte nossa de cada dia. Psicol Cienc Prof, 2002;22:30-41.

02. Society of Critical Care Medicine. Guidelines for ICU Admission, Discharge and Triage. Crit Care Med, 1999;27:633-638.

03. Benatti MC, Nishide VM - Development and implementation of an environmental risk map for the prevention of occupational accidents in an intensive care unit at a university hospital. Rev Lat Am Enfermagem, 2000;8:13-20

04. Nishide VM, Benatti MC-Occupational risks among a nursing staff working in an intensive care unit Rev Esc Enferm USP, 2004;38:406-414.
05. Gomes JR - Saúde ocupacional no hospital. Rev Paul Hosp, 1974;22:274-276.

06. Franco AR - Estudo preliminar das repercussões do processo de trabalho sobre a saúde dos trabalhadores de um hospital geral. Ribeirão Preto, 1981;217.

07. Silva VEF - Estudo sobre acidentes de trabalho ocorridos com trabalhadores de enfermagem de um hospital de ensino. São Paulo, 1988;176.

08. Alexandre NMC - Contribuição ao estudo das cervicodorsolombalgias em profissionais de enfermagem. Ribeirão Preto, 1993;185

09. Carrascal MSB - Infeccion VIH, transmisión horizontal. Rev Rol Enfermeria, 1991;14:60-62.

10. Machado AA, da Costa JC, Gir E et al - Risk of infections by the human immunodeficiency virus (HIV) among health professionals. Rev Saude Publica, 1992;26:54-56.

11. Pitta AMF - Hospital: Dor e Morte como Ofício. Sao Paulo: Hucitec, 1990.

12. US Public Health Service - Update U.S. Public Health Service Guidelines for the Management of Occupational Exposure to HBV, HCV, and HIV and Recommendations for Postexposure Prophylaxis. MMWR Recomm Rep, 2001:50(RR-11):1-52.

13. Resende MR, Fortaleza CMCB-Risco Ocupacional entre Profissionais da Área de Saúde e Medidas de Proteção, em: Colibrini MRC, Figueiredo RM, Paiva MC - Leito-dia em AIDS: Uma Experiência Multiprofissional. São Paulo: Atheneu; 2001;139-157.

14. Rapparini $\mathrm{C}$ - Riscos biológicos e profissionais de saúde: procedimentos clínicos. [online]. Disponível em http:/www. Risco biológico.org/riscos risc_procclinicos.htm. (27/8/2007).

15. Ministério do Trabalho - Normas Regulamentadoras: Segurança e Medicina do Trabalho. 48 Ed, São Paulo: Atlas, 2001.

16. Benatti MCC - Acidente do trabalho em um hospital universitário: um estudo sobre a ocorrência e os fatores de risco entre trabalhadores de enfermagem. São Paulo, 1997.

17. Costa MNA, Deus IA - Riscos ocupacionais em UTI: proteção específica. Rev Bras Enferm, 1989;42:106-109.

18. National Institute for Occupational Safety and Health - Guidelines for protecting the safety and health care workers [online]. Atlanta; 1988. Disponível em: http:// www.cdc.gov/ niosh/hcwold1.html (27/8/2007).

19. Funden - El Riesgo Professional, em: Manual de Salud Laboral. Madrid; 1996;93-8. (Serie enfermería).

20. L'Abbate $\mathrm{S}$, Smeke ELM, Oshiro JH - A educação em saúde como um exercício de cidadania. Saúde em Debate, 1992;37:81-85.

21. Rosso CFW, Collet $\mathrm{N}$ - Os enfermeiros e a prática de educação em saúde em município do interior paranaense. Rev Eletr Enferm, 1999;1:

22. Schall VT, Struchiner M - Educação no Contexto da Epidemia de HIV/ Aids: Teorias e Tendências Pedagógicas, em: Czeresnia D - Aids, Pesquisa Social e Educação, São Paulo: Editora Hucitec, Rio de Janeiro: Abrasco, 1995;84-105.

23. Moritz RD - Dilemas éticos sobre o fim da vida. Rev Bras Ter Intensiva 2003;15:3-4.

24. Coutrin RMGS, Freua PR, Guimarães CM - Estresse em enfermagem: uma análise do conhecimento produzido na literatura brasileira no período de 1982 a 2001. Texto \& Contexto Enferm, 2003;12:486-494

25. Santos JM, Oliveira EB, Moreira AC - Estresse, fator de risco para a saúde do enfermeiro em centro de terapia intensiva. Rev Enferm UERJ, 2006;14:580-585

26. Gomes GC, Lunardi Filho WD, Erdmann AL - O sofrimento psíquico em trabalhadores de UTI interferindo no seu modo de viver a enfermagem. Rev Enferm UERJ, 2006;14:93-99.

27. Savoldi NAM - Condições de Trabalho e Saúde dos Trabalhadores de Enfermagem da UTI Pediátrica. Rio de Janeiro; 2004;136.

28. Souto MC - CTI Oncológico: As Experiências do Trabalhador de Enfermagem com os Riscos, Sofrimento e Prazer. Rio de Janeiro; 2003; XVIII,110.

29. Gámez Perales M, Santano Magariño A, Matías Benayas S et al - Study of the work climate in four critical care units in a hospital. Enferm Intensiva, 1999;10:120-128.

30. Teegen F, Müller J - Trauma exposure and post-traumatic stress disorder in intensive care unit personnel. Psychother Psychosom Med Psychol, 2000;50:384-390

31. Hays MA, All AC, Mannahan C et al. - Reported stressors and ways of coping utilized by intensive care unit nurses. Dimens Crit Care Nurs, 2006;25:185-193. 
32. Caine RM; Ter-Bagdasarian $L$ - Early identification and management of critical incident stress. Crit Care Nurse, 2003;23:59-65.

33. Lynch J, Appelboam R, McQuillan PJ - Survey of abuse and violence by patients and relatives towards intensive care staff. Anaesthesia, 2003;58:893-899.

34. Early MR - Recognizing and managing violence in the NICU. Neonatal Netw, 2004;23:31-34

35. Drury T - Recognizing the potential for violence in the ICU. Dimens Crit Care Nurs, 1997;16:314-323.

36. Santos WDF - Acidentes típicos de trabalho em pessoal de enfermagem: fatores associados. Rev Bras Saúde Ocup, 1989;17:38-42.

37. Souza M, Vianna LAC - Incidência de acidentes de trabalho relacionada com a não utilização das precauções universais. Rev Bras Enfermagem, 1993;46:234-244.

38. Silva A - Estudo sobre os acidentes de trabalho ocorridos com a equipe de enfermagem em unidade de centro cirúrgico de um hospital geral. São Paulo. Congresso Brasileiro de Enfermagem em Centro Cirúrgico, São Paulo, 1995. Anais. São Paulo: Sociedade Brasileira de Enfermagem de Centro Cirúrgico, 1995;94-102.

39. Ministério da Saúde - Recomendações para atendimento e acompanhamento de exposição ocupacional a material biológico. HIV e hepatites $B$ e C. Brasília, 2004.

40. Stone PW, Gershon RR - Nurse work environments and occupational safety in intensive care units. Policy Polit Nurs Pract, 2006;7:240-247.

41. Lamontagne F, Abiteboul D, Lolom I et al - Role of safety-engineered devices in preventing needlestick injuries in 32 French hospitals. Infect Control Hosp Epidemiol, 2007;28:18-23.

42. de Oliveira BR, Murofuse NT - Occupational accidents and occupational disease: study of the hospital workers' knowledge about health risks of their work. Rev Lat Am Enfermagem, 2001;9:109-115.

43. Gallasch, $\mathrm{CH}$, Alexandre NMC - Avaliação dos riscos ergonômicos durante a movimentação e transporte de pacientes em diferentes unidades hospitalares. Rev Enferm UERJ, 2003;11:252-260.

44. Stucke S, Menzel NN - Ergonomic assessment of a critical care unit. Crit Care Nurs Clin North Am, 2007;19:155-165.
45. Hignett S, Lu J - Evaluation of critical care space requirements for three frequent and high-risk tasks. Crit Care Nurs Clin North Am, 2007;19:167-175.

46. Mostafa G, Sing RF, McKeown R et al - The hazard of scattered radiation in a trauma intensive care unit. Crit Care Med, 2002;30:574-576.

47. Flor Rde C, Kirchhof AL - An educative practice of sensitization to ionizing radiation exposition with health professionals. Rev Bras Enfermagem, 2006;59:274-278.

48. Carvalho WB, Pedreira ML, de Aguiar MA - Noise level in a pediatric intensive care unit. J Pediatr, 2005;81:495-498.

49. Tsiou C, Eftymiatos D, Theodossopoulou E et al - Noise sources and levels in the Evgenidion Hospital intensive care unit. Intensive Care Med, 1998;24:845-847.

50. Morrison WE, Haas EC, Shaffner DH et al - Noise, stress, and annoyance in a pediatric intensive care unit. Crit Care Med, 2003;31:113-119.

51. Hilgemberg CE, Pusch SR, Youssef $\mathrm{N}$ et al - Um estressor denominado "Ruído". X Congresso Brasileiro de Terapia Intensiva. Rev Bras Ter Intensiva, 2002;(Suppl).

52. Christensen $\mathrm{M}$ - What knowledge do ICU nurses have with regard to the effects of noise exposure in the Intensive Care Unit? Intensive Crit Care Nurs, 2005;21:199-207.

53. Philbin MK, Gray $L-$ Changing levels of quiet in an intensive care nursery. J Perinatol, 2002;22:455-460.

54. Giampaoli E - Temperaturas Extremas, em: Astete MW, Giampaoli E, Zidan LN - Riscos físicos. São Paulo: Fundacentro, 1985;33-99.

55. Guidelines for intensive care unit design. Guidelines/Practice Parameters Committee of the American College of Critical Care Medicine. Society of Crit Care Medicine, Crit Care Med, 1995;23:582-588.

56. Barbosa A - Riscos químicos ocupacionais em hospitais da Fundação Hospitalar do Distrito Federal (FHDF). Rev Saude DF, 1990;1:8-15.

57. Forrester BG, Roth VS - Hand dermatitis in intensive care units. J Occup Environ Med, 1998;40:881-885.

58. Patrícia JC, María AL, Mônica CP - Percepción de riesgo laboral de las enfermeras de una unidad de cuidado intensivo. Cienc Enferm, 2001;7:57-67. 